Supplement of Atmos. Chem. Phys., 16, 4785-4797, 2016

http://www.atmos-chem-phys.net/16/4785/2016/

doi:10.5194/acp-16-4785-2016-supplement

(C) Author(s) 2016. CC Attribution 3.0 License.

(c) (i)

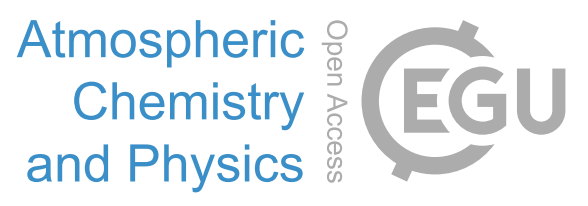

Supplement of

\title{
Introduction: Observations and Modeling of the Green Ocean Amazon (GoAmazon2014/5)
}

\section{S. T. Martin et al.}

Correspondence to: S. T. Martin (scot_martin@harvard.edu)

The copyright of individual parts of the supplement might differ from the CC-BY 3.0 licence. 


\section{List of Supplementary Tables}

Table S1. Instrumentation deployed at T0a (ATTO) during the Intensive Operating Periods of GoAmazon2014/5.

Table S2. Instrumentation deployed at T0e (EMPRAPA) during the Intensive Operating Periods of GoAmazon2014/5.

Table S3. Instrumentation deployed at T0k (K34; ZF2) during the Intensive Operating Periods of GoAmazon2014/5.

Table S4. Instrumentation deployed at T0t (TT34; ZF2) during the Intensive Operating Periods of GoAmazon2014/5.

Table S5. Instrumentation deployed at T1p (Ponta Pelada) during the Intensive Operating Periods of GoAmazon2014/5.

Table S6. Instrumentation deployed at T1 (INPA campus) during the Intensive Operating Periods of GoAmazon2014/5.

Table S7. Instrumentation deployed at T2 (Tiwa) during the Intensive Operating Periods of GoAmazon2014/5.

Table S8. University instrumentation deployed at T3 (Fazenda Agropecuária Exata) during the Intensive Operating Periods of GoAmazon2014/5.

Table S9. MAOS instrumentation deployed at T3 (Fazenda Agropecuária Exata) during the Intensive Operating Periods of GoAmazon2014/5.

Table S10. AMF-1 instrumentation deployed at T3 (Fazenda Agropecuária Exata) during the Intensive Operating Periods of GoAmazon2014/5.

Table S11. Instrumentation deployed at T3u (UEA) during the Intensive Operating Periods of GoAmazon2014/5. 
Table S12. Instrumentation deployed on board the G1 (AMF) aircraft during the Intensive Operating Periods of GoAmazon2014/5.

Table S13. Instrumentation deployed on board the HALO aircraft during the second Intensive Operating Period of GoAmazon2014/5. 


\section{List of Supplementary Figures}

Figure S1. Nighttime illumination in the environs of the city of Manaus $\left(-3.1^{\circ},-60.0^{\circ}\right)$ for the year 2010. The yellow points show the locations of the GoAmazon2014/5 research sites of Figure 1. Source: http://maps.ngdc.noaa.gov/viewers/dmsp gcv4/ and http://ngdc.noaa.gov/eog/dmsp/downloadV4composites.html, accessed 12 August 2015.

Figure S2. Flight tracks of the G1 aircraft during IOP1 segregated by (a) 250 to $750 \mathrm{~m}$ above sea level, (b) 750 to $1250 \mathrm{~m}$, (c) 1250 to $1750 \mathrm{~m}$, and (d) above $1750 \mathrm{~m}$.

Figure S3. Flight tracks of the G1 aircraft during IOP2 segregated by (a) 250 to $900 \mathrm{~m}$ above sea level, (b) 1000 to $2000 \mathrm{~m}$, and (c) above $2000 \mathrm{~m}$.

Figure S4. Locations of power plants in the environs of Manaus (orange pins). The location of a large refinery is also shown (blue pin). Yellow pins show the locations of some of the GoAmazon2014/5 research sites (cf. Figure 1). Source: personal communication, Adan Medeiros, 10 August 2015.

Figure S5. Locations of brick factories between Manaus (T1) and to the north of Manacapuru (T3). Source: personal communication, Bruno Portela, 8 September 2015.

Figure S6. Anomalies of sea surface temperature (SST) during (a) IOP1 and (b) IOP2. The climatology of 1981 through 2010 is the used as the reference for presentation of the anomalies in 2014 based on the NCEP/NCAR reanalysis. Source: http://rda.ucar.edu/datasets/ds090.0/, accessed 12 September 2015.

Figure S7. Satellite-based detection of fires on regional scale. Left and right columns represent periods of IOP1 and IOP2, respectively. Each fire icon indicates the detection of one fire event during the seven-day observation period. The yellow markers 
indicate the locations of GoAmazon2014/5 research sites (cf. Figure 1). Source: http://www.dpi.inpe.br/proarco/bdqueimadas/, accessed 7 August 2015.

Figure S8. Satellite-based detection of fires on the scale of South America. Left and right columns represent periods of IOP1 and IOP2, respectively. Color scaling in red runs from 0 to 100 fire events detected in a $1^{\circ} \times 1^{\circ}$ grid box during the seven-day observation period. Some grid boxes have more than 100 events. The green point represents the location of Manaus. Source:

http://www.dpi.inpe.br/proarco/bdqueimadas/, accessed 7 August 2015.

Figure S9. Aerosol optical depth observed by the MODIS instrument on the AQUA satellite (eight-day composites, Collection 5). Left and right columns represent periods of IOP1 and IOP2, respectively. Color scaling is from an optical depth of 0.0 to 0.8 . Black indicates regions over which optical depth was not retrieved, largely because of the high albedo of the Earth's surface in these regions. Source: http://modisatmos.gsfc.nasa.gov/MYD08 E3/browse c51.html, accessed 5 August 2015. 


\begin{tabular}{|c|c|c|c|c|}
\hline \multirow[b]{2}{*}{ Instrument } & \multirow[b]{2}{*}{ Abbreviation } & \multicolumn{2}{|c|}{ Data Available } & \multirow[b]{2}{*}{ Investigator } \\
\hline & & IOP1 & IOP2 & \\
\hline Aerosol Chemical Speciation Monitor (Aerodyne) & ACSM & Yes & Yes & Artaxo \\
\hline Scanning Mobility Particle Sizer (TSI) & SMPS & Yes & Yes & Artaxo \\
\hline Condensation Particle Counter (Grimm, model 5412) & СРC & Yes & Yes & Pöhlker \\
\hline Multiangle Absorption Photometer 5012 (Thermo, model 5012) & MAAP & Yes & Yes & Pöhlker \\
\hline Aethalometer (Magee) & AE30 & Yes & Yes & Artaxo \\
\hline Nephelometer (Ecotech Aurora 3000) & & Yes & Yes & Artaxo \\
\hline Ultra-High Sensitivity Aerosol Spectrometer (DMT) & UHSAS & Yes & Yes & Pöhlker \\
\hline Single Particle Soot Photometer (DMT) & SP2 & Yes & Yes & Pöhlker \\
\hline Size-Resolved Cloud Condesation Nuclei Counter (DMT) & Size-resolved CCNC & No & Yes & Artaxo/Pöhlker \\
\hline Optical Particle Sizer (TSI, model 3330) & OPS & Yes & Yes & Pöhlker \\
\hline Wide Range Aerosol Spectrometer (Grimm) & WRAS & No & Yes & Pöhlker \\
\hline Wideband Integrated Bioaerosol Spectrometer (DMT) & WIBS-4 & No & Yes & $\mathrm{Su}$ \\
\hline Proton-Trasfer-Reaction Quadrupole Mass Spectrometer (Ionicon) & PTR-Q-MS & Yes & Yes & Kesselmeier \\
\hline Picarro CRDS (Model G1302; S/N CKADS-018) & & Yes & Yes & Lavric \\
\hline Picarro CRDS (Model G1301; S/N CKADS-109) & & Yes & Yes & Lavric \\
\hline Chemolimuniscence NOx monitor (Ecotech CLD TR 780) & CLD & Yes & Yes & Sörgel \\
\hline $\mathrm{CO} 2$ monitor (LI-COR 7000) & & Yes & Yes & Sörgel \\
\hline Ozone monitor (TEI 49 I) & & Yes & Yes & Sörgel / Souza \\
\hline Optical Fog Sensor (Eigenbrodt) & & No & Yes & Pöhlker \\
\hline Soil Heat Flux Sensor (HFP01, Hukseflux) & & Yes & Yes & Araujo \\
\hline Water Content Reflector (CS615, Campbell) & & Yes & Yes & Araujo \\
\hline Thermistor (108, Campbell) & & Yes & Yes & Araujo \\
\hline Pyranometer (CMP21 \& CMP4, Kipp \& Zonen) & & Yes & Yes & Araujo \\
\hline Quantum Sensor (PAR-LITE, Kipp \& Zonen) & & Yes & Yes & Araujo \\
\hline Net radiometer (NR-LITE, Kipp \& Zonen) & & Yes & Yes & Araujo \\
\hline UV radiometer (Kipp \& Zonen, CUV5) & & Yes & Yes & Araujo \\
\hline Rain Gauge (TB4, Hydrological Services Pty. Ltd.) & & Yes & Yes & Araujo \\
\hline Termohygrometer (CS215, Rotronic Measurement Solutions) & & Yes & Yes & Araujo \\
\hline Barometer (PTB101B, Vaisala) & & Yes & Yes & Araujo \\
\hline 2-D Sonic Anemometer (Windsonic, Gill) & & Yes & Yes & Araujo \\
\hline 3-D Sonic Anemometer (Windmaster, Gill) & & Yes & Yes & Araujo \\
\hline IRGA (LI-7500A \& LI7200, LI-COR) & & Yes & Yes & Araujo \\
\hline
\end{tabular}




\begin{tabular}{llcll}
\hline & & & Data Available & \\
Instrument & Abbreviation & IOP1 & IOP2 & Investigator \\
\hline Hg Monitor (TEKRAN) & Tekran & Yes & Yes & Artaxo \\
Nephelometer (Ecotech) & Neph_M9003 & Yes & Yes & Artaxo \\
Multi filter shadow band radiometer (YesInc) & MFR & Yes & Yes & Barbosa \\
Sun Photometer (Cimel) & Aeronet & Yes & Yes & Artaxo \\
UV Raman Lidar (Raymetrics) & Lidar & Yes & Yes & Barbosa \\
24GHz Micro Rain Radar (Metek) & MRR & No & Yes & Barbosa \\
Laser precipitation monitor (Thies) & Disdro_Thies & Yes & Yes & Barbosa \\
Meteorological weather station (Davis) & MetDavis & No & No & Barbosa / Souza \\
Meteorological weather station (Thies) & MetThies & No & Yes & Barbosa \\
Meteorological weather station (Gill) & MetGill & No & Yes & Barbosa \\
NetR8 GNSS Receiver (Trimble) & GNSS & Yes & Yes & Barbosa \\
Precipitation Collector NSA 181/KE DURAN (Eigenbrodt) & Eigenbrodt & No & Yes & Barbosa \\
Meteorological weather station (Campbell) & MetCambpell & Yes & Yes & Machado \\
Passive Infraread Microwave radiometer MP3000 (Radiometrics) & MP3000 & Yes & No & Machado \\
Parsivel Disdrometer (OTT) & Parsivel & Yes & No & Machado \\
IRGASON Integrated CO2 and H2O Open-Path Gas Analyzer and 3D Sonic Anem. FluxCampbell & Yes & Yes & Machado \\
\hline
\end{tabular}




\begin{tabular}{llccl}
\hline & & & Data Available & \\
Instrument & Abbreviation & IOP1 & IOP2 & Investigator \\
\hline Proton tranfer reaction mass spectrometer & PTR-MS & Yes & Yes & Jardine \\
Gas chromatograph - mass spectrometter & GC-MS & Yes & Yes & Jardine \\
Proton tranfer reaction mass spectrometer & PTR-MS & No & Yes & Chamecki/Fuentes \\
Gas chromatograph - mass spectrometter & GC-MS & No & Yes & Chamecki/Fuentes \\
Sonic anemometers (10) & & No & Yes & Chamecki/Fuentes \\
Water vapor gas analyzer & IRGA & No & Yes & Chamecki/Fuentes \\
Carbon dioxide gas analyzer & IRGA & No & Yes & Chamecki/Fuentes \\
Ozone gas analyzer & & No & Yes & Chamecki/Fuentes \\
Sulfur dioxide gas analyzer & & No & Yes & Chamecki/Fuentes \\
Carbon monoxide gas analyzer & & No & Yes & Chamecki/Fuentes \\
Nitric oxide gas analyzer & & No & Yes & Chamecki/Fuentes \\
Fast mobility particle sizer & FMPS & No & Yes & Chamecki/Fuentes \\
Cloud condensation nuclei counter & CCN counter & No & Yes & Chamecki/Fuentes \\
Picarro (CO, CO2, H2O) & CKADS & Yes & Yes & Jardine/Fuentes \\
\hline
\end{tabular}




\begin{tabular}{llccc}
\hline & & & Data Available \\
Instrument & Abbreviation & IOP1 & IOP2 & Investigator \\
\hline High-Resolution Time-of-Flight Aerosol Mass Spectrometer (Aerodyne Inc.) & HR-ToF-AMS & Yes & No & McFiggans \\
Proton-Trasfer-Reaction Quadrupole Mass Spectrometer (Ionicon) & PTR-Q-MS & Yes & No & McFiggans \\
Multiangle Absorption Photometer 5012 (Thermo) & MAAP & Yes & Yes & Artaxo \\
Nephelometer (TSI) & & Yes & Yes & Artaxo \\
Tapered-Element Oscillating Microbalance & TEOM & No & Yes & Artaxo \\
Picarro (CO, CO2, $\mathrm{H} 2 \mathrm{O})$ & CKADS & Yes & Yes & Artaxo \\
Ozone & Thermo_49i & Yes & Yes & Artaxo \\
Four daily sondes at $\left(-2.639^{\circ},-60.157^{\circ}\right)(7.5 \mathrm{~km}$ to the southeast of T0t) & & Yes & Yes & Machado \\
\hline
\end{tabular}


$\bar{D} \equiv \bar{E}$ 


$\begin{array}{ccc}\text { Instrument } & \text { Abbreviation } & \text { Data Available } \\ \text { IOP1 } & \text { IOP2 }\end{array}$

Four daily radio sondes

Abbreviation

$\begin{array}{ll}\text { DOP1 IOP2 } & \text { IO A } \\ \text { OPI }\end{array}$

S-band radar operated by the Amazon Protection System (SIPAM) 


\begin{tabular}{llccc}
\hline & & Data Available & \\
Instrument & Abbreviation & IOP1 & IOP2 & Investigator \\
\hline Proton-Trasfer-Reaction Quadrupole Mass Spectrometer (Ionicon) & PTR-Q-MS & Yes & Yes & Artaxo \\
Ozone Monitor 49i (Thermo) & O3 Monitor & Yes & Yes & Artaxo \\
SO2 Monitor 43i (Thermo) & SO2 Monitor & Yes & Yes & Artaxo \\
Cavity Attenuated Phase Shift NO2 Monitor (Aerodyne Inc.) & CAPS NO2 & No & Yes & Artaxo \\
CO, N2O monitor (Los Gatos Research) & CO, NO2 LGR & Yes & Yes & Souza \\
Scanning Mobility Particle Sizer (TSI Inc.) & SMPS & Yes & Yes & Artaxo \\
Aerosol Chemical Speciation Monitor (Aerodyne Inc.) & ACSM & Yes & Yes & Artaxo \\
Multiangle Absorption Photometer 5012 (Thermo) & MAAP & Yes & Yes & Artaxo \\
Aethalometer (Magee Inc.) & 71- AE33 & Yes & Yes & Artaxo \\
Nephelometer (Ecotech) & 3-1 Neph Aurora & Yes & Yes & Artaxo \\
Tapered Element Oscillating Microbalance 1405 (Thermo) & TEOM PM2.5 \& PM10 & No & Yes & Artaxo \\
Cloud Condensartion Nuclei Counter (Droplet Measurement Technologies) & Size-resolved CCNC & No & Yes & Artaxo \\
\hline
\end{tabular}




\begin{tabular}{|c|c|c|c|c|}
\hline \multirow[b]{2}{*}{ Instrument } & \multirow[b]{2}{*}{ Abbreviation } & \multicolumn{2}{|c|}{ Data Available } & \multirow[b]{2}{*}{ Investigator } \\
\hline & & IOP1 & IOP2 & \\
\hline Disdrometer (Parsivel) & & Yes & Yes & Machado \\
\hline X-Band Radar & & Yes & Yes & Machado \\
\hline Micro Rain Radar & MRR & No & Yes & Machado \\
\hline High-Resolution Time-of-Flight Aerosol Mass Spectrometer (Aerodyne Inc.) & HR-ToF-AMS & Yes & Yes & Alexander/Martin \\
\hline Semi-Volatile Thermal Desorption Aerosol Gas Chromatograph & SV-TAG & Yes & Yes & Goldstein \\
\hline Size-Resolved Cloud Condensation Nuclei Counter & SCCN & Yes & Yes & Wang \\
\hline Harvard Rebound Apparatus & HBA & Yes & Yes & Martin \\
\hline Oxidation Flow Reactor & OFR & Yes & Yes & Jimenez \\
\hline Proton Transfer Reaction Time-of-Flight Mass Spectrometer & PTR-ToF-MS & Yes & Yes & Martin/MAOS \\
\hline Proton Transfer Reaction Time-of-Flight Mass Spectrometer & PTR-ToF-MS & Yes & Yes & Guenther \\
\hline Scanning Mobility Particle Sizer & SMPS & Yes & Yes & Jimenez \\
\hline HOx Chemical Ionization Mass Spectrometer & HOx-CIMS & Yes & Yes & Kim \\
\hline Picarro CRDS G2401 analyzer (CO, CO2, CH4) & Picarro & Yes & Yes & Jimenez \\
\hline Medium Volume Filter Sampler, PM1 (MCV, S.A.) & & Yes & Yes & Jimenez \\
\hline Air Quality Monitoring $60(\mathrm{O} 3)$ & AQM-60 & Yes & Yes & Souza \\
\hline Sequential Filter Sampler & & Yes & Yes & Goldstein \\
\hline Transmission electron microscope grip sampler & TEM grid sampler & Yes & Yes & Buseck \\
\hline PM2.5 Filter Sampler & & Yes & Yes & Godoi/Souza \\
\hline Filter sampler (quartz and nuclepore) & & Yes & Yes & Artaxo \\
\hline Laser-induced flurescence & LIF & Yes & Yes & Keutsch \\
\hline Micro-Orifice Uniform-Deposit Impactor & MOUDI & No & Yes & Laskin \\
\hline Neutral cluster and Air Ion Spectrometer & NAIS & No & Yes & Petaja \\
\hline Nano particle size distribution & NPSD & Yes & Yes & Kuang \\
\hline Passive tubes for trace gases & & Yes & Yes & Godoi/Souza \\
\hline Particle Size Magnifier & PSM & No & Yes & Petaja \\
\hline Laser Induced Phosphorescence & LIP & No & Yes & Keutsch \\
\hline Thermal Desorption Chemical Ionization Mass Spectrometer & TD-CIMS & Yes & Yes & Smith \\
\hline Precipication collection for chemical analysis & & No & Yes & Pauliquevis \\
\hline Solar radiation and photosynthenitically active radiation & & Yes & Yes & Souza \\
\hline Meteorological station & & Yes & Yes & Souza \\
\hline
\end{tabular}




\begin{tabular}{llcc}
\hline Instrument & Abbreviation & \multicolumn{2}{c}{ Data Available } \\
IOP1 & IOP2 \\
\hline Aerosol Chemistry Speciation Monitor & ACSM & No & Yes \\
Aethalometer, 7-Wavelength & Aeth & Yes & Yes \\
CO, N2O and H2O Analyzer & CO/N2O/H2O & Yes & Yes \\
Condensation Particle Counter, Fine Mode & CPCf & Yes & Yes \\
Condensation Particle Counter, Ultrafine Mode & CPCu & No & No \\
Meteorology Sensor & Met & Yes & Yes \\
Nephelometer & Neph & Yes & Yes \\
Nephelometer, dry & NephDry & Yes & Yes \\
Oxides of Nitrogen Analyzer & NO/NOx/NOy & Yes* & Yes \\
Ozone Analyzer & O3 & Yes & Yes \\
Photoacoustic Soot Spectrometer, 3-Wavelength & PASS-3 & No & No \\
Particle Soot Absorption Photometer & PSAP & Yes & Yes \\
Proton Transfer Mass Spectrometer & PTRMS & Yes & Yes \\
Scanning Mobility Particle Sizer & SMPS & No & No \\
Single Particle Soot Photometer & SP2 & Yes & Yes \\
SO2 Analyzer & SO2 & No & Yes \\
Ultra-High Sensitivity Aerosol Spectrometer & UHSAS & Yes & Yes \\
\hline
\end{tabular}




\begin{tabular}{|c|c|c|c|}
\hline \multirow[b]{2}{*}{ Instrument } & \multirow[b]{2}{*}{ Abbreviation } & \multicolumn{2}{|c|}{ Data Available } \\
\hline & & IOP1 & IOP2 \\
\hline Two-Channel Narrow Field of View Zenith Radiometer & $2 \mathrm{NFOV}$ & Yes & No \\
\hline Atmospheric Emitted Radiance Interferometer & AERI & Yes & Yes \\
\hline Propeller Vane Wind Sensors & Anemometer & Yes & Yes \\
\hline Black and White pyranometer, shaded, downwelling & B/W 8-48-SKY & Yes & Yes \\
\hline Barometer & Barometer & Yes & Yes \\
\hline Ceilometer Cloud Lidar & Ceilometer & Yes & Yes \\
\hline Cloud Condensation Nuclei Counter & $\mathrm{CCN}$ & Yes & Yes \\
\hline Continuous Light Absorption Photometer & CLAP & Yes & No \\
\hline Condensation Particle Counter & $\mathrm{CPC}$ & Yes & Yes \\
\hline Cimel Sunphotometer & CSPHOT & Yes & No \\
\hline Doppler Lidar & $\mathrm{DL}$ & Yes & Yes \\
\hline Eddy Correlation Flux Measurement System & ECOR & No & Yes \\
\hline Infrared Thermometer Downlooking & IR Therm Grd & Yes & Yes \\
\hline Infrared Thermometer Uplooking & IRT-Sky & Yes & Yes \\
\hline ARM-Standard Meteorological Instrumentation at Surface & Met & Yes & Yes \\
\hline Multi-Filter Radiometer & MFR & No & Yes \\
\hline Multi-Filter Rotating Shadow Band Radiometer & MFRSR & Yes & Yes \\
\hline Micropulse Lidar & MPL & No & Yes \\
\hline Microwave Radiometer & MWR & Yes & Yes \\
\hline Microwave Radiometer 3 Channel & MWR 3C & Yes & Yes \\
\hline Microwave Radiometer High Frequency & MWR HF & No & No \\
\hline Microwave Radiometer P & MWR P & No & No \\
\hline Nephelometer & Neph & Yes & Yes \\
\hline Nephelometer, wet & NephDry & No & Yes \\
\hline Normally Incident Pyroheliometer: Installed and recording data & NIP & Yes & Yes \\
\hline Optical Rain Gauge & ORG & No & No \\
\hline Parsivel Present Weather detector & Parsivel2 disdrometer & No & No \\
\hline Pyrogeometer, upwelling & PIR-GRND & Yes & Yes \\
\hline Pyrogeometer, downwelling & PIR-SKY & Yes & Yes \\
\hline Present Weather Detector & PWD & Yes & Yes \\
\hline Particle Soot Absorption Photometer & PSAP & Yes & Yes \\
\hline Precision Spectral Pyranometer, upwelling & PSP-GRND & Yes & Yes \\
\hline Precision Spectral Pyranometer, downwelling & PSP-SKY & Yes & Yes \\
\hline Radar Wind Profiler & RWP & Yes & Yes \\
\hline Ka/W-band Scanning ARM Cloud Radar & SACR & No & No \\
\hline Shortwave Array Spectroradiometer - $180 \mathrm{deg}$ field of view & SAS-HE & Yes & Yes \\
\hline Shortwave Array Spectroradiometer - Zenith pointing & SAS-ZE & Yes & Yes \\
\hline Surface Energy Balance System & SEBS & Yes & No \\
\hline Sound-based wind profiler & SODAR & Yes & Yes \\
\hline Sonic Anemometer & Sonic & No & Yes \\
\hline Tipping Bucket Rain Gauge & TB Rain Gauge & No & No \\
\hline Total Sky Imager & TSI & No & No \\
\hline Temperature and Humidity Sensor & $\mathrm{T} / \mathrm{RH}$ & Yes & Yes \\
\hline W-band (95 GHz) ARM Cloud Radar & WACR & Yes & Yes \\
\hline Five daily radio sondes $(0,6,12,15$ and 18 UTC) & & Yes & Yes \\
\hline
\end{tabular}




\begin{tabular}{lcccc}
\hline & & & & Data Available \\
Instrument & Abbreviation & IOP1 & IOP2 & Investigator \\
\hline Disdrometer (Joss) & & Yes & Yes & Machado \\
Disdrometer (Parsivel) & & Yes & Yes & Machado \\
Rain Gauge & & Yes & Yes & Machado \\
Microwave Radiometer (MP3000) & MRR & No & Yes & Machado \\
Micro Rain Radar & IWV & Yes & Yes & Machado \\
GPS-Based Integrated Water Vapor & & Yes & Yes & Machado \\
Field Mill & & Yes & Yes & Machado \\
\hline
\end{tabular}




\begin{tabular}{|c|c|c|c|}
\hline \multirow[b]{2}{*}{ Instrument } & \multirow[b]{2}{*}{ Abbreviation } & \multicolumn{2}{|c|}{ Data Available } \\
\hline & & IOP1 & IOP2 \\
\hline Global Positioning System (DSM 232) & GPS & Yes & Yes \\
\hline Temperature (Rosemount E102AL) & & Yes & Yes \\
\hline Pressure (Rosemount 1201F1) & & Yes & Yes \\
\hline Gust Probe: Rosemount 1221F2 (3 units) & & Yes & Yes \\
\hline Chilled-mirror hygrometer (GE-1011B) & & Yes & Yes \\
\hline Tuneable Diode Laser Hygrometer & TDL-H & Yes & Yes \\
\hline Aircraft Integrated Meteorological Measurement System & AIMMS-20 & Yes & Yes \\
\hline Video Camera P1347 (forward looking) & & Yes & Yes \\
\hline Video Camera P1344 & & Yes & Yes \\
\hline Ultrafine Condensation Particle Counter (Model 3025A) & $\mathrm{UCPC}$ & Yes & Yes \\
\hline Condensation Particle Counter (Model 3010) & $\mathrm{CPC}$ & Yes & Yes \\
\hline Fast Integrated Mobility Spectrometer & FIMS & Yes & Yes \\
\hline Ultra High Sensitivity Aerosol Spectrometer - Airborne & UHSAS-A & Yes & Yes \\
\hline Passive Cavity Aerosol Spectrometer & PCASP & Yes & Yes \\
\hline Particle/Soot Absorption Photometer & PSAP & Yes & Yes \\
\hline Three-Wavelength Integrating Nephelometer (Model 3563) & & Yes & Yes \\
\hline High Resolution Aerosol Mass Spectrometer & HR-ToF-AMS & Yes & Yes \\
\hline Cloud Condensation Nuclei Counter & $\mathrm{CCN}$ Counter & Yes & Yes \\
\hline Optical Particle Counter (Model CI-3100) & $\mathrm{OPC}$ & Yes & Yes \\
\hline Quadrupole Proton-Transfer-Reaction Mass Spectrometer & PTR-MS & Yes & Yes \\
\hline $\mathrm{N} 2 \mathrm{O}$ and $\mathrm{CO}$ concentrations (Los Gatos $23 \mathrm{r}$ ) & & Yes & Yes \\
\hline Oxides of Nitrogen (Nox) & & Yes & Yes \\
\hline Ozone (Thermo Scientific Model 49i) & & Yes & Yes \\
\hline Picarro Cavity Ring Down System (Model G1301-m) & CRD & Yes & Yes \\
\hline Multi Element Water content System (SEA WCM-2000) & & Yes & Yes \\
\hline High Volume Precipitation Spectrometer (Version 3) & HVPS-3 & Yes & Yes \\
\hline Two-Dimensional Stereo Probe & 2D-S & Yes & Yes \\
\hline Fast Cloud Droplet Probe & Fast-CDP & Yes & Yes \\
\hline Cloud Droplet Probe (version 2) & CDP ver2 & Yes & Yes \\
\hline Cloud Particle Imager (version 2) & CPI ver2 & Yes & Yes \\
\hline Sunshine Pyranometer, shaded & SPN-1 & Yes & Yes \\
\hline Sunshine Pyranometer, unshaded (up and down looking) & SPN-1 & Yes & Yes \\
\hline
\end{tabular}




\begin{tabular}{|c|c|c|c|}
\hline \multirow[b]{2}{*}{ Instrument } & \multirow[b]{2}{*}{ Abbreviation } & \multicolumn{2}{|c|}{ Data Available } \\
\hline & & & IOP2 \\
\hline $\begin{array}{l}\text { Counter-flow Virtual Impactor Inlet } \\
\end{array}$ & CVI inlet & No & Yes \\
\hline Precipitation Imaging Probe & PIP & No & Yes \\
\hline Cloud Combination Probe (Cloud ImagingProbe + Cloud Droplet Probe) & $\mathrm{CCP}(\mathrm{CIP}+\mathrm{CDP})$ & No & Yes \\
\hline Novel Ice EXpEriment - Cloud and Aerosol Particle Spectrometer & NIXE-CAPS & No & Yes \\
\hline Small Ice Detector Probe (version 3) & SID-3 & No & Yes \\
\hline Microwave Temperature Profiler & MTP & No & Yes \\
\hline Ultra High Sensitivity Aerosol Spectrometer - Airborne & UHSAS-A & No & Yes \\
\hline Cloud and Aerosol Spectrometer with Depolarization & CAS-DPOL & No & Yes \\
\hline Particle Habit Imaging and Polar Scattering Probe & PHIPS-HALO & No & Yes \\
\hline Compact Time of Flight Aerosol Mass Spectrometer & C-ToF-AMS & No & Yes \\
\hline Aerosol MEasuremenT SYSTem & AMESTYST & No & Yes \\
\hline SiNgle-ParticleSOOtPhotometer System & SNOOPY & No & Yes \\
\hline HALO Aerosol Submicrometer Inlet & HASI & No & Yes \\
\hline Cloud Condensation Nucleus Counter & $\mathrm{CCNC}$ & No & Yes \\
\hline Photo-Acoustic Spectrometer & PAS & No & Yes \\
\hline Single Particle Soot Photometer & SP-2 & No & Yes \\
\hline Fast Ice Nucleus Chamber & FINCH & No & Yes \\
\hline $\mathrm{CO}, \mathrm{O} 3$ & AMTEX & No & Yes \\
\hline $\mathrm{SO} 2, \mathrm{HNO}, \mathrm{PFC}$ & PAN-MS & No & Yes \\
\hline $\mathrm{NOy}, \mathrm{NO}$ & IPA-NOy & No & Yes \\
\hline Mini Differential Optical Absorption Spectroscopy & miniDOAS & No & Yes \\
\hline Spectral Modular Airborne Radiation Measurement System & SMART & No & Yes \\
\hline Imaging Spectrometer Package Eagle/Hawk & & No & Yes \\
\hline Hygrometer for Atmospheric Investigations & HAI & No & Yes \\
\hline
\end{tabular}




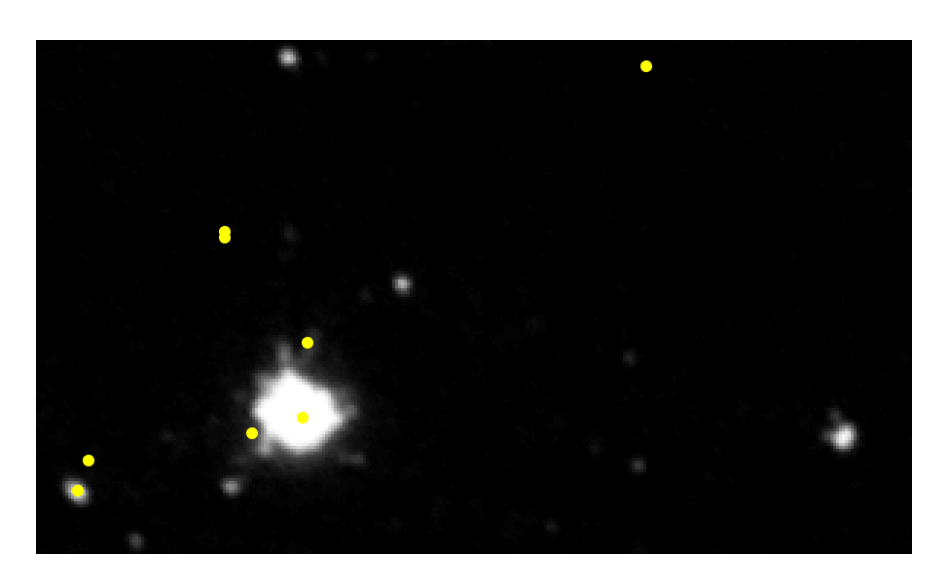


a. IOP1 G1 FlightTrajectories $250 \mathrm{~m}$ to $750 \mathrm{~m}$

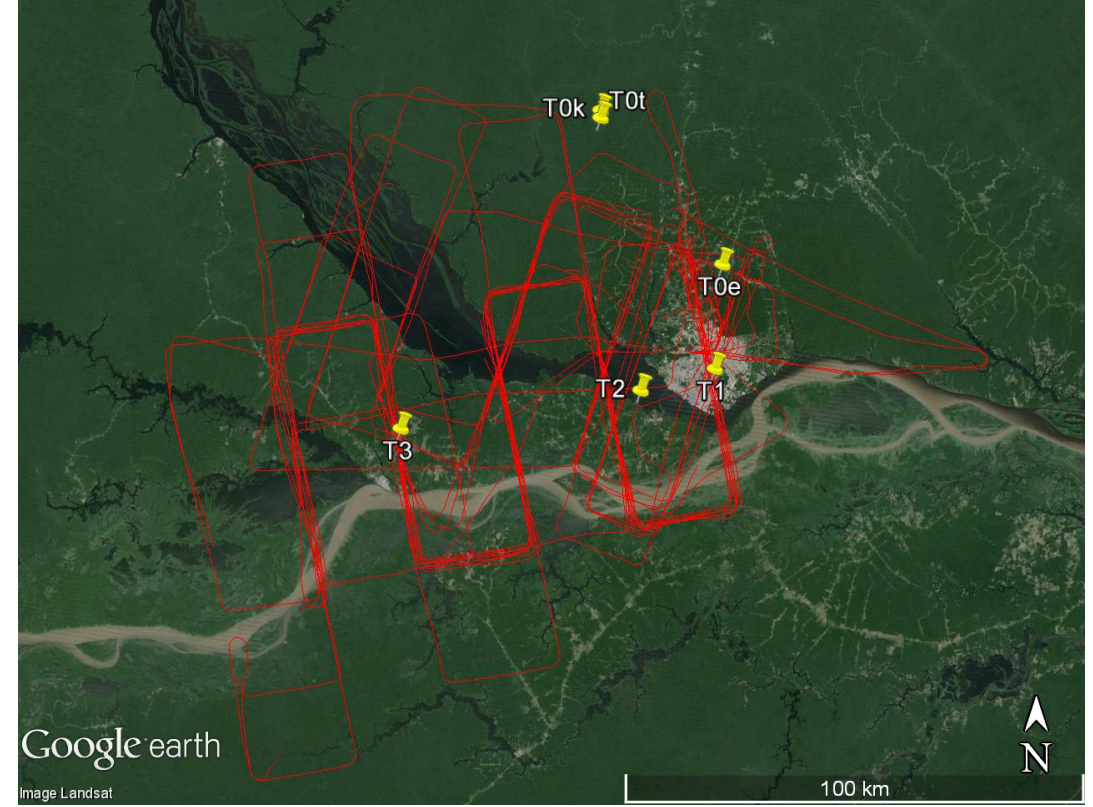

\section{b. IOP1 G1 FlightTrajectories $750 \mathrm{~m}$ to $1250 \mathrm{~m}$}

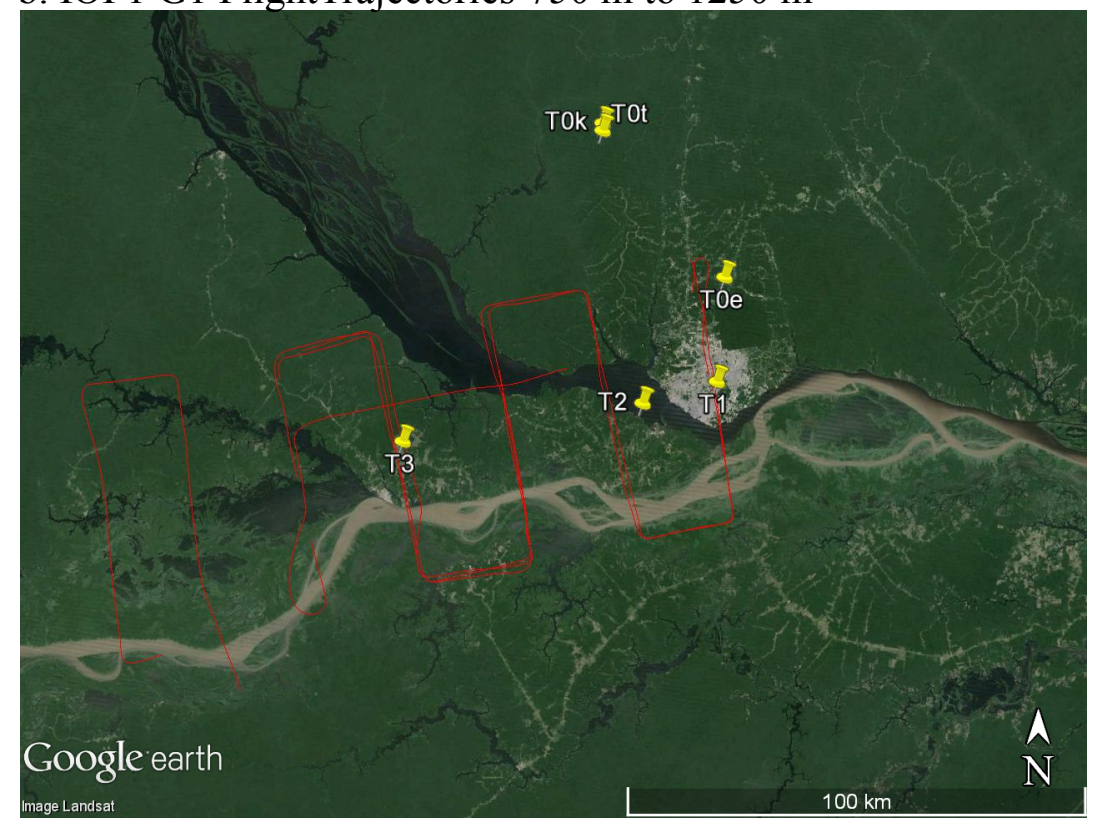

c. IOP1 G1 FlightTrajectories $1250 \mathrm{~m}$ to $1750 \mathrm{~m}$

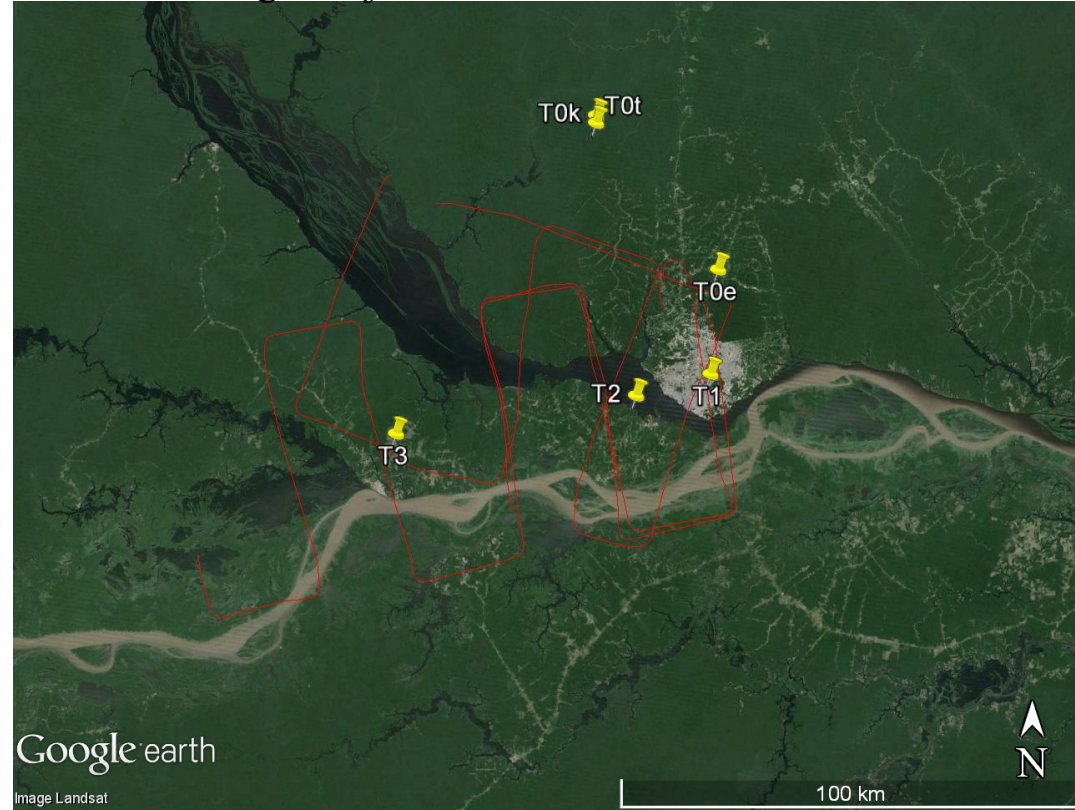

d. IOP1 G1 FlightTrajectories above $1750 \mathrm{~m}$

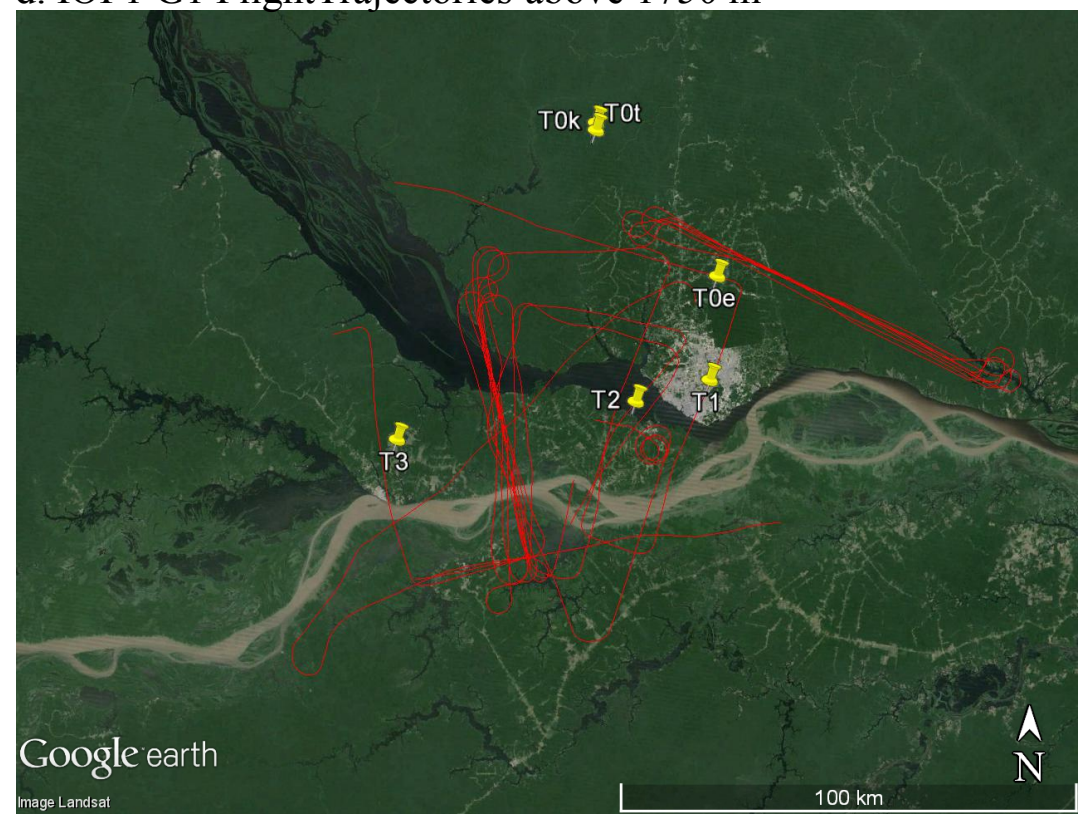

Figure S2 
a. IOP2 G1 FlightTrajectories $250 \mathrm{~m}$ to $900 \mathrm{~m}$

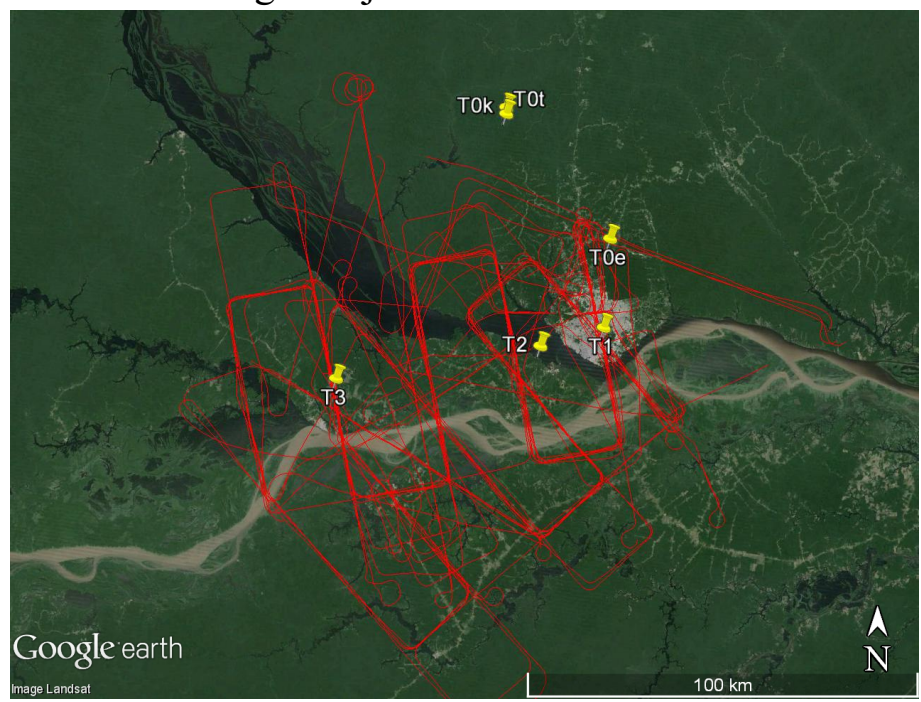

b. IOP2 G1 FlightTrajectories $1000 \mathrm{~m}$ to $2000 \mathrm{~m}$

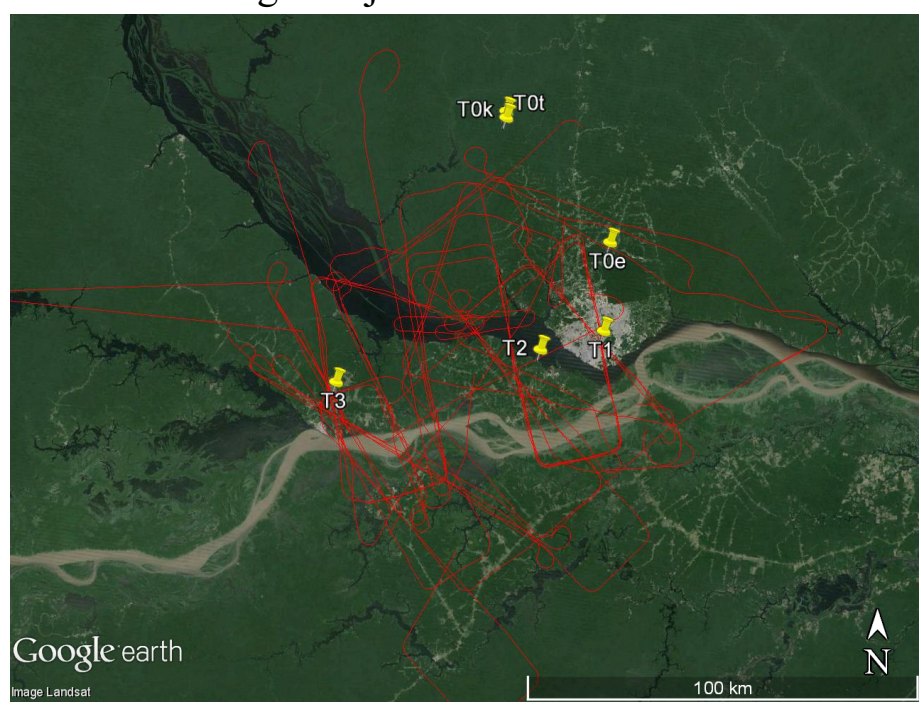

c. IOP2 G1 FlightTrajectories above $2000 \mathrm{~m}$

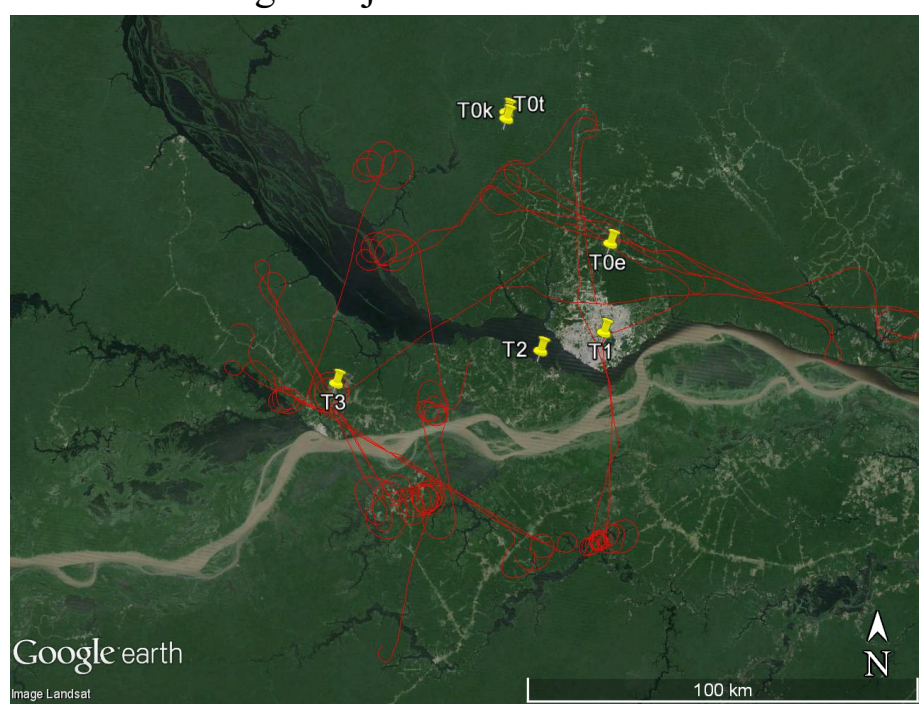

Figure S3 


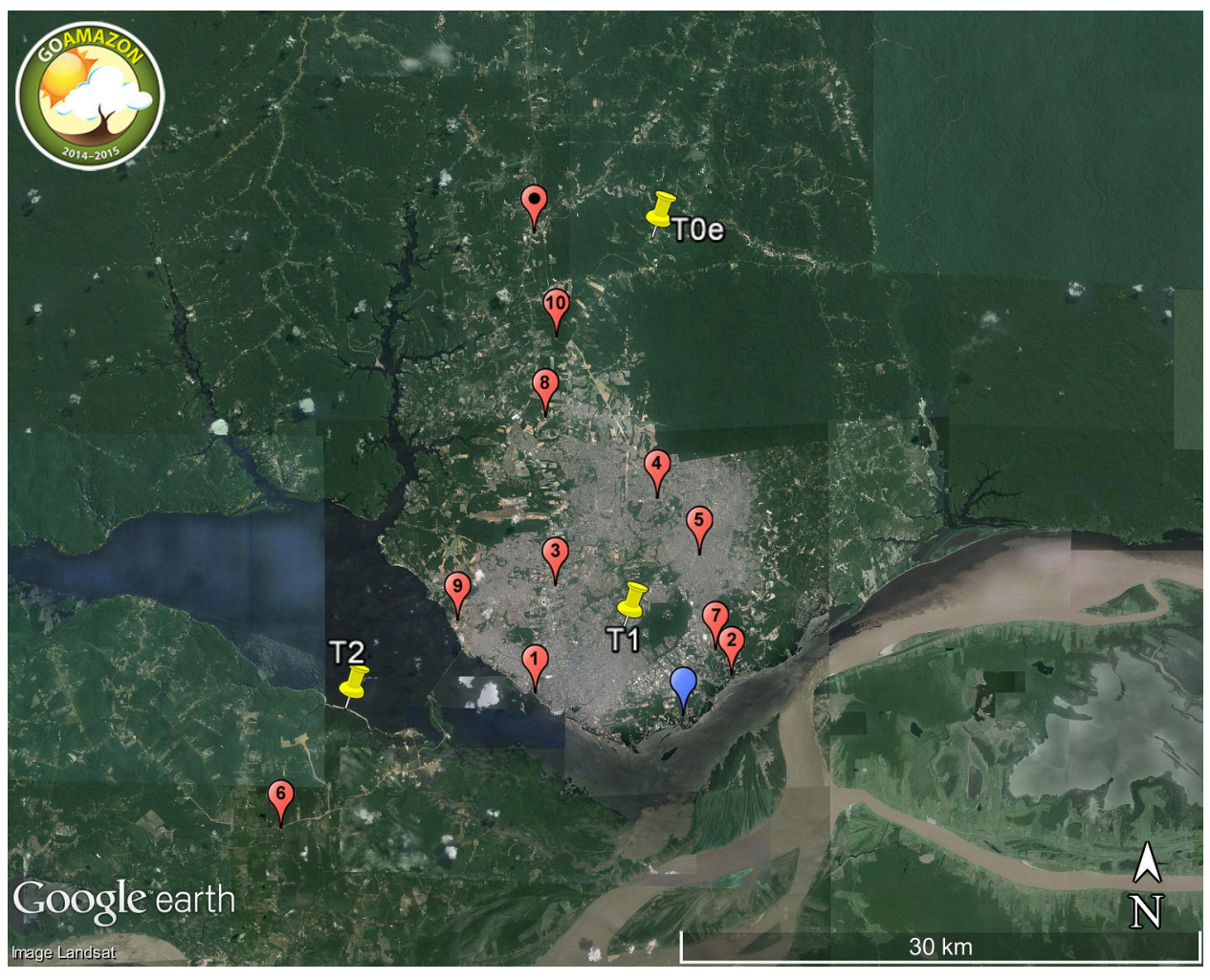

Figure S4 


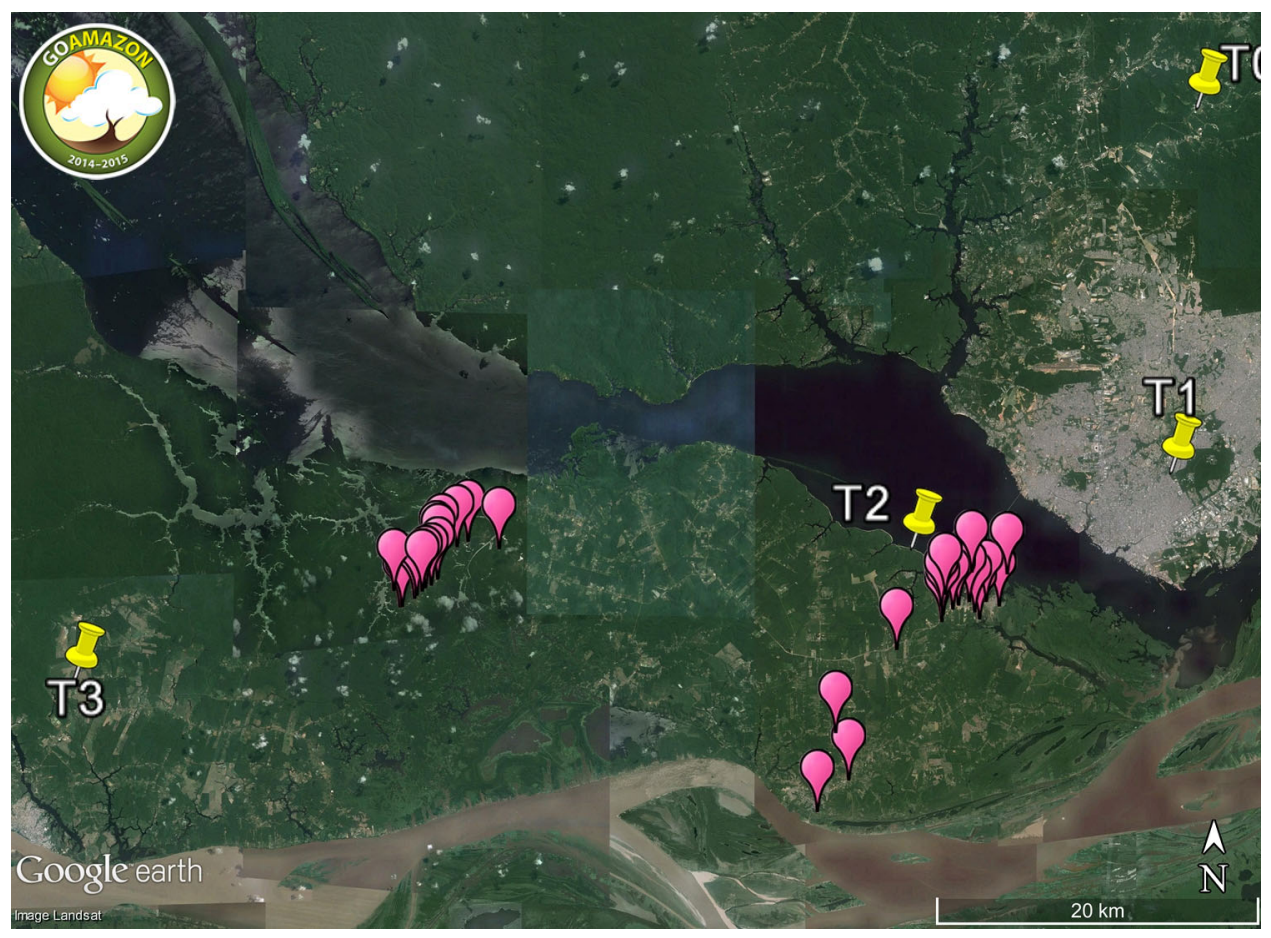

Figure S5 


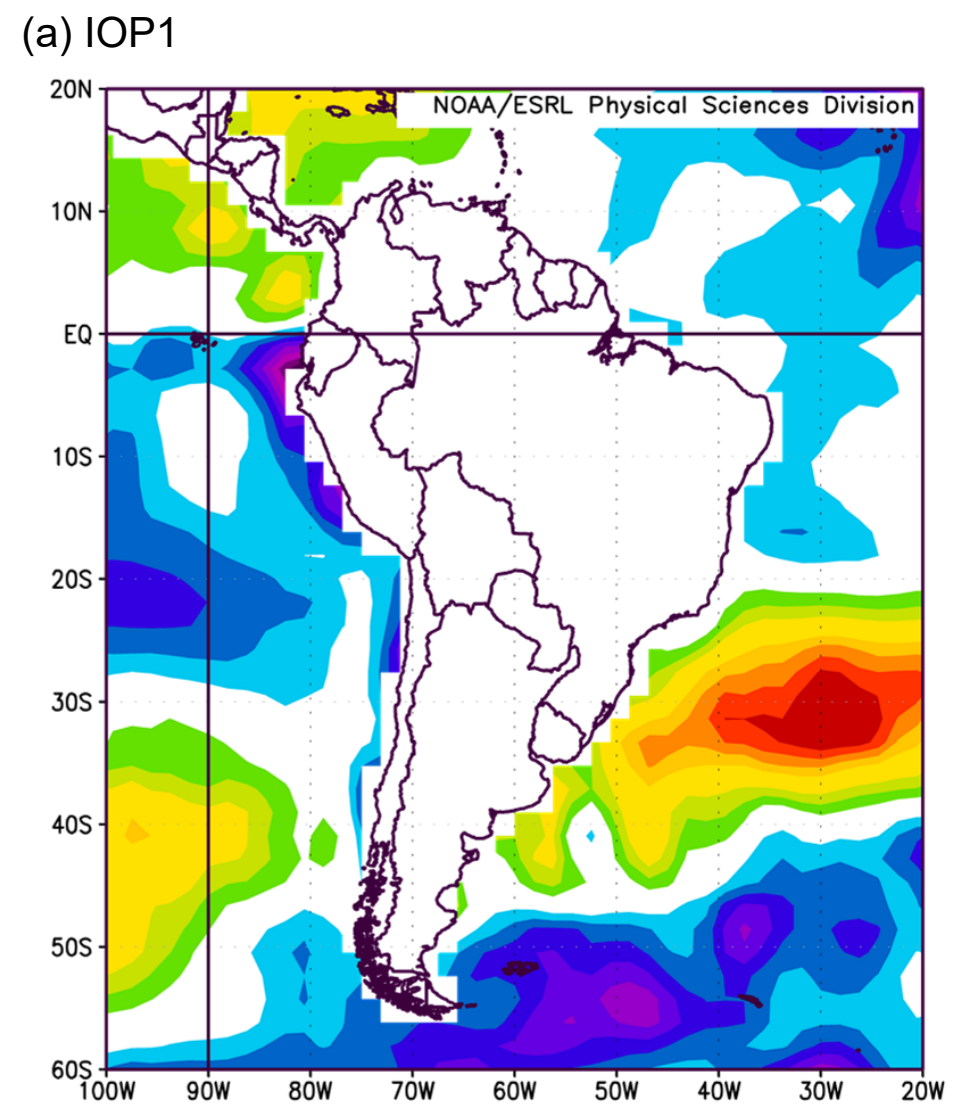

(b) IOP2
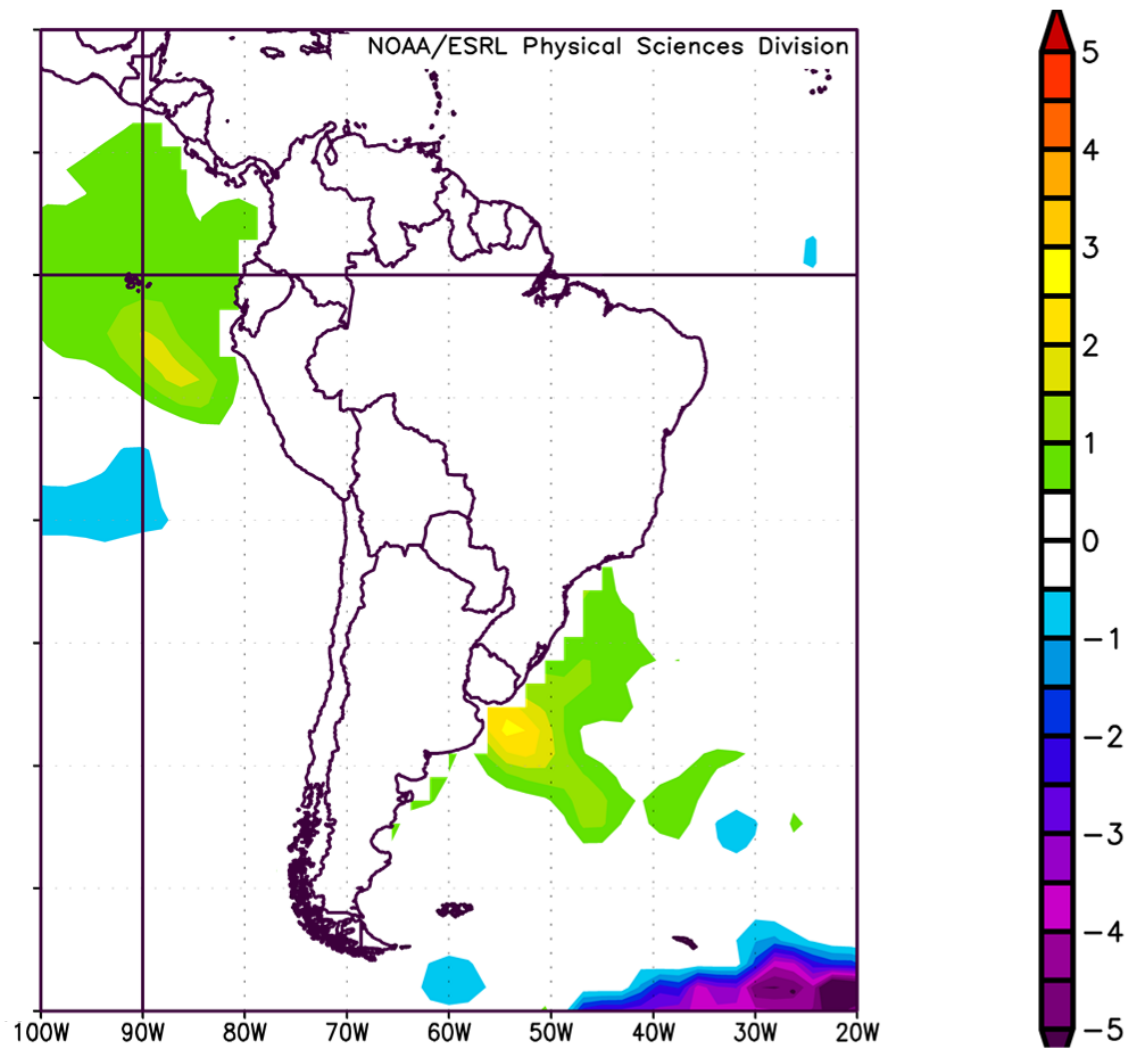


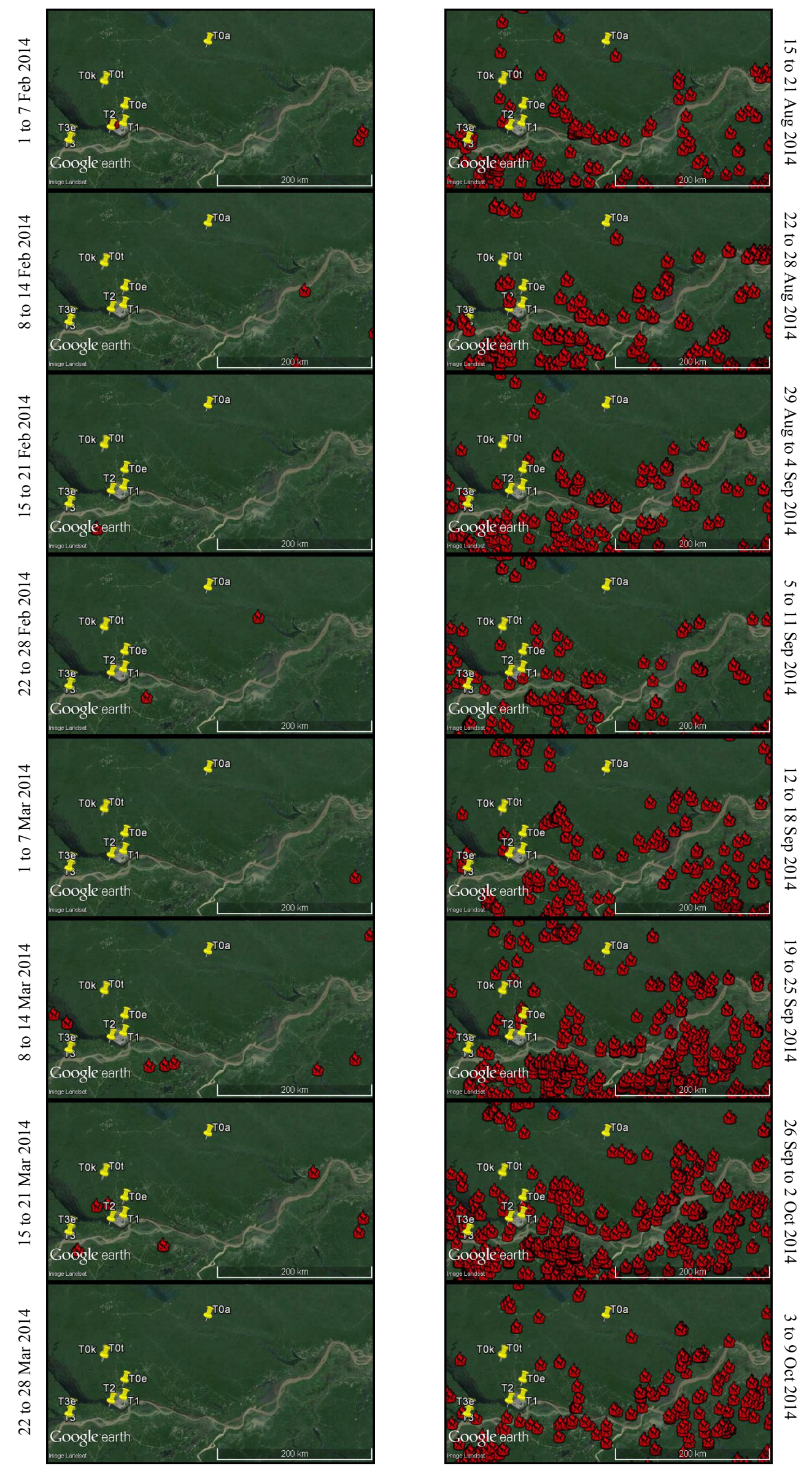

Figure S7 

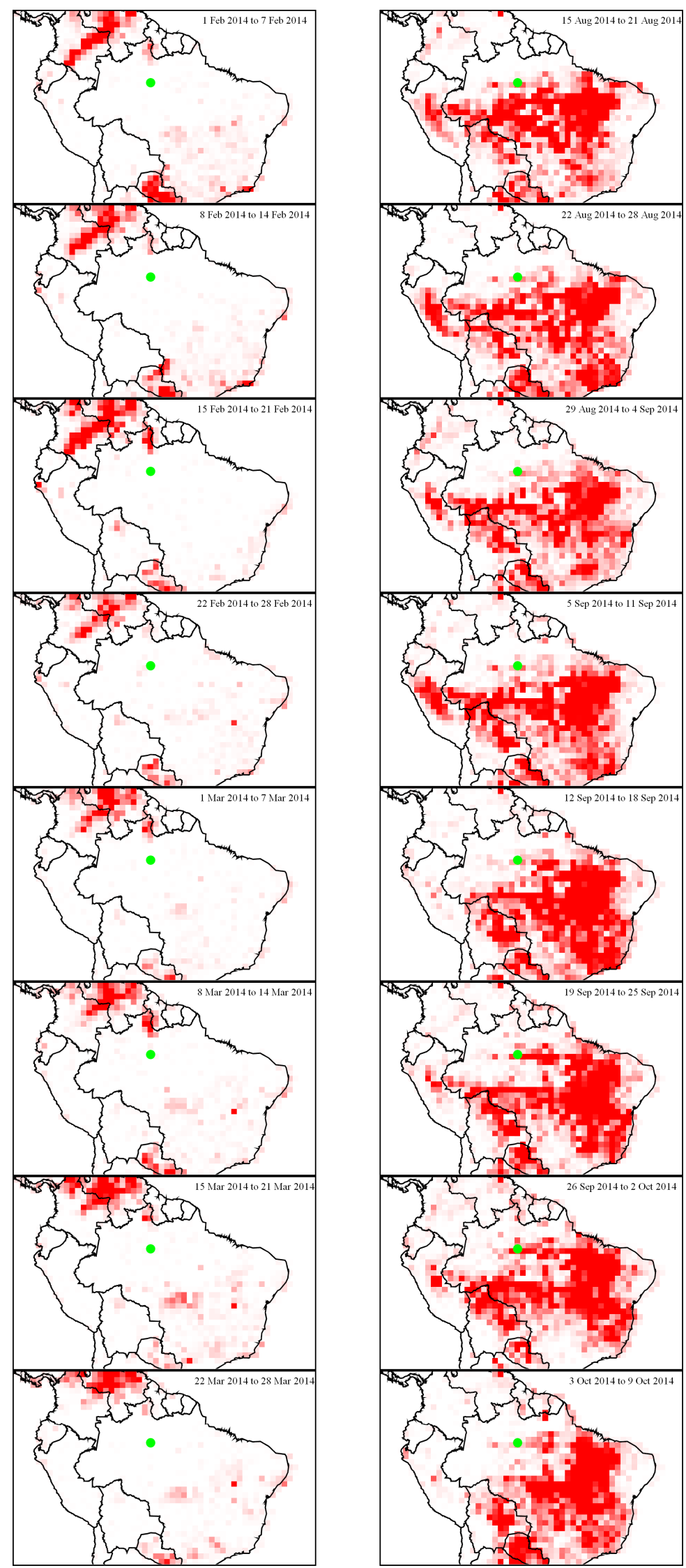

Figure S8 

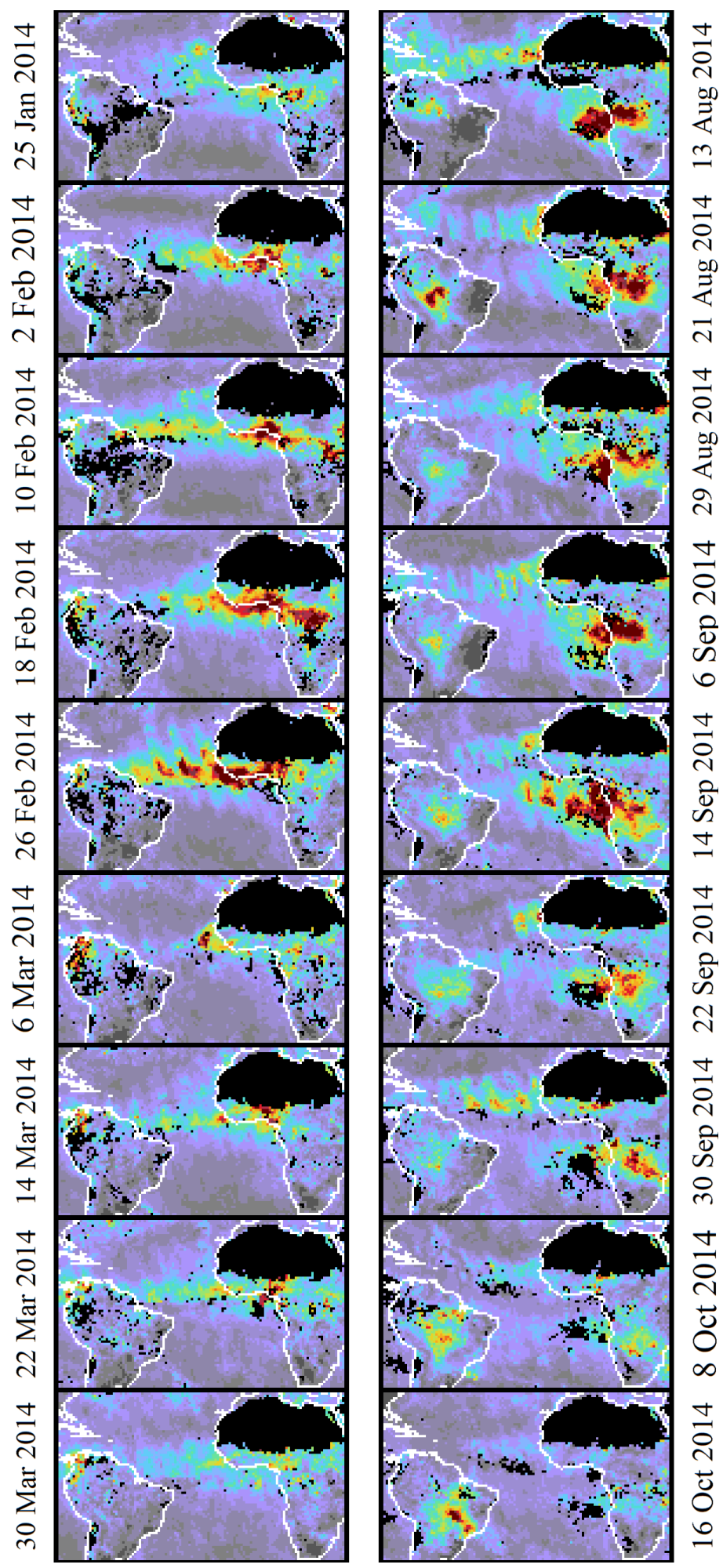

Figure S9 